\title{
Investigation of intravalence, core-valence and core-core electron correlation effects in polonium atomic structure calculations
}

\author{
Pascal Quinet ${ }^{\mathrm{a}, \mathrm{b}, *}$ \\ a Astrophysique et Spectroscopie, Université de Mons, B-7000 Mons, Belgium \\ ${ }^{\mathrm{b}}$ IPNAS, Université de Liège, B-4000 Liège, Belgium
}

\section{A R T I C L E I N F O}

\section{Article history:}

Received 23 December 2013

Received in revised form

29 April 2014

Accepted 1 May 2014

Available online 10 May 2014

Keywords:

Atomic structure

Radiatve atomic data

Hartree-Fock atomic structure calculations

\begin{abstract}
A B S T R A C T
A detailed investigation of the atomic structure and radiative parameters involving the lowest states within the $6 p^{4}, 6 p^{3} 6 d, 6 p^{3} 7 s, 6 p^{3} 7 p$ and $6 p^{3} 7 d$ configurations of neutral polonium is reported in the present paper. Using different physical models based on the pseudo-relativistic Hartree-Fock approach, the influence of intravalence, core-valence and core-core electron correlation on the atomic parameters is discussed in detail. This work allowed us to fix the spectroscopic designation of some experimental level energy values and to provide for the first time a set of reliable oscillator strengths corresponding to 31 Po I spectral lines in the wavelength region from 175 to $987 \mathrm{~nm}$.
\end{abstract}

(c) 2014 Elsevier Ltd. All rights reserved.

\section{Introduction}

Because of the high radioactivity of all its isotopes, polonium $(Z=84)$ is a very rare element in nature. According to Fry and Thoennessen [1], forty-two polonium isotopes with atomic masses ranging from $A=186$ to $A=227$ have been discovered so far. All of these isotopes are characterized by short half-lives, the most stable species being ${ }^{209}$ Po with a half-life of 102 years. Among naturally occuring polonium, ${ }^{210}$ Po has the longest half-life with 138.39 days. This isotope can be found in small concentrations in the earth's crust and in the atmosphere. Nevertheless, despite its rarity in our environment, this chemical element arouses a growing interest since a few years because of its potential applications in different scientific fields such as nuclear physics and astrophysics.

\footnotetext{
* Correspondence address: Astrophysique et Spectroscopie, Université de Mons, B-7000 Mons, Belgium.

E-mail address: quinet@umons.ac.be
}

In nuclear physics, the spectroscopic properties of polonium has attracted much attention over the last years. In particular, laser-induced fluorescence spectroscopy in a collimated atomic beam was applied by Kowalewska and coworkers [2] to determine isotope shifts and the hyperfine structure of the isotopic chain ${ }^{200} \mathrm{Po},{ }^{202} \mathrm{Po},{ }^{204-210} \mathrm{Po}$. The measurements were based on the observation of resonance fluorescence after excitation of the atomic transition $6 \mathrm{p}^{4}{ }^{3} \mathrm{P}_{2} \rightarrow 6 \mathrm{p}^{3} 7 \mathrm{~s}{ }^{5} \mathrm{~S}_{2}$ of Po I. More recently, three new ionization schemes for polonium were tested with the resonant ionization laser ion source (RILIS) during the online production of ${ }^{196} \mathrm{Po}$ in a $\mathrm{UC}_{x}$ target at CERN-ISOLDE. The saturation of the atomic transitions were observed and the yields of the isotope chain ${ }^{193-198,200,202,204}$ Po were measured [3]. With the same facility, in-source resonant ionization laser spectroscopy of the even- $A$ polonium isotopes ${ }^{192-210,216,218}$ Po was performed using the $6 \mathrm{p}^{3} 7 \mathrm{~s}$ ${ }^{5} \mathrm{~S}_{2} \rightarrow 6 \mathrm{p}^{3} 7 \mathrm{p}{ }^{5} \mathrm{P}_{2}$ transition in Po I [4]. In this latter work, the comparison of the measured isotope shifts in ${ }^{200-210} \mathrm{Po}$ with previous data set allowed the authors to test for the first time large-scale atomic calculations [5] used to extract the changes in the mean-square charge radius 
of the atomic nucleus. The same experiment was also used to extract new information on the $\beta$ decay and the hyperfine structure of ${ }^{199}$ Po [6]. As support to further experimental or theoretical studies of nuclear properties in polonium, it is essential to know the atomic structure of this element with the highest possible accuracy.

In astrophysics, recent observations have suggested the presence of short-lived radioactive elements, such as Tc ( $Z=43)$, Pm $(Z=61)$ and $84 \leq Z \leq 99$ elements at the surface of the chemically peculiar roAp star HD 101065, also known as Przybylski's star [7-10]. In particular, Gopka et al. [8] presented the results on new identifications of the lines of all radioactive elements with atomic numbers from $Z=84$ to $Z=99$, except for $Z=85$ (At) and $Z=87$ (Fr). The presence of these heavy short-lived radioactive elements in HD 101065 star is enigmatic (see discussion in [7-13]). Goriely [14] has stressed the importance of spallation nucleosynthesis compared to diffusion processes as a possible explanation of the chemical composition of the outer layers of some chemically peculiar stars. The available astrophysical spectroscopic observations are however affected by large uncertainties essentially due to the lack of atomic data for the elements of interest.

These heavy elements are also an interesting challenge for the theoretical calculations in view of their very complex atomic structures containing a large number of electrons. In the present work, we concentrate on the particular case of neutral polonium (Po I) for which different pseudo-relativistic Hartree-Fock (HFR) models have been used to compute the atomic structure and radiative parameters involving the lowest states within the $6 p^{4}, 6 p^{3} 6 d, 6 p^{3} 7 s, 6 p^{3} 7 p$ and $6 p^{3} 7 d$ configurations. This study is an extension of our previous theoretical works dedicated to the atomic properties of some other short-lived radioelements such as technecium $(Z=43)$ [15], promethium $(Z=61)$ [16], francium $(Z=87)$ [17], radium $(Z=88)$ [18], actinium $(Z=89)$ [18] and americium $(Z=95)[19]$.

\section{The experimental spectrum and energy levels of polonium}

In the current atomic spectra database available on the National Institute of Standards and Technology (NIST) website [20], 32 energy level values of Po I ranging from 0 to $64451 \mathrm{~cm}^{-1}$ are listed and classified as belonging to $6 p^{4}, 6 p^{3} 7 p, 6 p^{3} 8 p, 6 p^{3} 9 p, 6 p^{3} 10 p$ and $6 p^{3} 6 d, 6 p^{3} 7 d, 6 p^{3} 8 d$, $6 p^{3} 7 s, 6 p^{3} 8 s$ even-parity and odd-parity configurations, respectively. These values were essentially taken from the work of Charles [21] who revised and extended the previous experimental analyses of the polonium spectrum performed by Charles et al. [22] and Mrozowski [23]. More precisely, Charles [21] reported the wavelengths of 97 lines of ${ }^{208} \mathrm{Po}$ and the hyperfine structure of 31 lines of ${ }^{209} \mathrm{Po}$ covering the spectral range $210-900 \mathrm{~nm}$ obtained by measurement of Fabry-Perot interferograms using a radiation source containing a sample of polonium composed of 33 micrograms of ${ }^{208}$ Po and 11 micrograms of ${ }^{209}$ Po. From these data, relative values of 26 energy levels of ${ }^{208} \mathrm{Po}$ I were deduced. However, for about half of those levels, the author was unable to give a spectroscopic designation or even a total angular momentum $J$-quantum number with certainty. As an example, the three lowest energy levels of $6 p^{3} 7 \mathrm{p}$ configuration appearing at 50681.28, 50934.89 and $51636.42 \mathrm{~cm}^{-1}$ were reported by Charles [21] with uncertain $J$-values. However, more recently, three new ionization schemes for polonium were tested with the resonant ionization laser ion source (RILIS) during the on-line production of ${ }^{196} \mathrm{Po}$ in a $\mathrm{UC}_{x}$ target at CERN-ISOLDE [3]. In the first ionization scheme, one valence electron was excited from the $6 \mathrm{p}^{4}{ }^{3} \mathrm{P}_{2}$ ground state with a UV transition at $255.8 \mathrm{~nm}$ to the $6 \mathrm{p}^{3} 7 \mathrm{~s}{ }^{5} \mathrm{~S}_{2}$ excited state. A second excited state was reached with an infrared transition at $843.38 \mathrm{~nm}$ before the electron occupying this level was excited to the continuum by the non-resonant absorption of a $510.6 \mathrm{~nm}$ photon, provided by a Cu vapor laser. During this experiment, the resonance curve of the second step was observed confirming the existence of the excited state at $50934.89 \mathrm{~cm}^{-1}$ with $6 \mathrm{p}^{3}\left({ }^{4} \mathrm{~S}\right) 7 \mathrm{p}{ }^{5} \mathrm{P}_{2}$ as spectroscopic designation.

\section{Atomic structure calculations}

The computational procedure that we have used for modeling the atomic structure and calculating radiative parameters in Po I is the pseudo-relativistic Hartree-Fock method described in detail by Cowan [24]. In order to investigate the effects of intravalence, core-valence and core-core electron correlations, six sets of calculations (AF) were performed in the present work. These are summarized in Table 1. In view of the large number of uncertainties affecting the positions, the spectroscopic designations and/or the $J$-quantum numbers in the configurations with $n \geq 7$, we focused our study on the lowest states belonging to $6 p^{4}, 6 p^{3} 6 d, 6 p^{3} 7 s, 6 p^{3} 7 p$ and $6 p^{3} 7 d$ configurations. As seen from Table 1 , more and more electron correlation is progressively included when going from model A to model F. More precisely, in calculation A, only valence correlation outside $6 s^{2} 6 p^{3}$ is considered while some single and double excitations from the $6 \mathrm{p}$ subshell are included in calculations $B$ and $C$, respectively. In calculation $\mathrm{D}$, core-valence correlation effects are added by including single excitations from the 6 s subshell while, in calculation E, core-core electron correlation contributions are considered with double excitations from $6 \mathrm{~s}$. Finally, in model $\mathrm{F}$, the core-polarization (CPOL) effects from the 78 electrons occupying the $1 s^{2} 2 s^{2} 2 p^{6} 3 s^{2} 3 p^{6} 3 d^{10}$ $4 s^{2} 4 p^{6} 4 d^{10} 4 f^{14} 5 s^{2} 5 p^{6} 5 d^{10}$ closed shells corresponding to the Po VII ionic core were estimated using a pseudopotential and a correction to the dipole operator according to the HFR + CPOL procedure described in many of our previous papers (see e.g. [25-27]). These CPOL corrections depend on two paremeters, i.e. the electric dipole polarizability of the ionic core, $\alpha_{d}$, and the cut-off radius, $r_{c}$, which can be seen as a measure of the size of the ionic core. For the first parameter, a value of $\alpha_{d}=2.00 a_{0}^{3}$ was graphically extrapolated for Po VII from the theoretical values of Fraga et al. [28] along the platinum isoelectronic sequence (see Fig. 1). The cut-off radius, $r_{c}$, was chosen to be equal to $1.17 a_{0}$ which corresponds to the HFR average value $\langle r\rangle$ for the outermost core orbital (5d). 
Table 1

Interacting configurations and core-polarization parameters retained in the HFR atomic structure calculations of neutral polonium.

\begin{tabular}{|c|c|c|c|c|c|c|}
\hline Type of calculation & A & B & C & $\mathrm{D}$ & $\mathrm{E}$ & $\mathrm{F}$ \\
\hline Even configurations & $\begin{array}{l}6 s^{2} 6 p^{4} \\
6 s^{2} 6 p^{3} 7 p \\
6 s^{2} 6 p^{3} 5 f \\
6 s^{2} 6 p^{3} 6 f\end{array}$ & $\begin{array}{l}6 s^{2} 6 p^{4} \\
6 s^{2} 6 p^{3} 7 p \\
6 s^{2} 6 p^{3} 5 f \\
6 s^{2} 6 p^{3} 6 f \\
6 s^{2} 6 p^{2} 6 d^{2} \\
6 s^{2} 6 p^{2} 7 s^{2} \\
6 s^{2} 6 p^{2} 6 d 7 s \\
6 s^{2} 6 p^{2} 6 d 7 d \\
6 s^{2} 6 p^{2} 7 s 7 d \\
6 s^{2} 6 p^{2} 7 p 5 f \\
6 s^{2} 6 p^{2} 7 p 6 f\end{array}$ & $\begin{array}{l}6 s^{2} 6 p^{4} \\
6 s^{2} 6 p^{3} 7 p \\
6 s^{2} 6 p^{3} 5 f \\
6 s^{2} 6 p^{3} 6 f \\
6 s^{2} 6 p^{2} 6 d^{2} \\
6 s^{2} 6 p^{2} 7 s^{2} \\
6 s^{2} 6 p^{2} 6 d 7 s \\
6 s^{2} 6 p^{2} 6 d 7 d \\
6 s^{2} 6 p^{2} 7 s 7 d \\
6 s^{2} 6 p^{2} 7 p 5 f \\
6 s^{2} 6 p^{2} 7 p 6 f \\
6 s^{2} 6 p 7 p^{3} \\
6 s^{2} 6 p 6 d^{2} 7 p \\
6 s^{2} 6 p 7 s^{2} 7 p \\
6 s^{2} 6 p 6 d 7 s 7 p\end{array}$ & $\begin{array}{l}6 s^{2} 6 p^{4} \\
6 s^{2} 6 p^{3} 7 p \\
6 s^{2} 6 p^{3} 5 f \\
6 s^{2} 6 p^{3} 6 f \\
6 s^{2} 6 p^{2} 6 d^{2} \\
6 s^{2} 6 p^{2} 7 s^{2} \\
6 s^{2} 6 p^{2} 6 d 7 s \\
6 s^{2} 6 p^{2} 6 d 7 d \\
6 s^{2} 6 p^{2} 7 s 7 d \\
6 s^{2} 6 p^{2} 7 p 5 f \\
6 s^{2} 6 p^{2} 7 p 6 f \\
6 s^{2} 6 p 7 p^{3} \\
6 s^{2} 6 p 6 d^{2} 7 p \\
6 s^{2} 6 p 7 s^{2} 7 p \\
6 s^{2} 6 p 6 d 7 s 7 p \\
6 s 6 p^{4} 6 d \\
6 s 6 p^{4} 7 s \\
6 s 6 p^{3} 6 d 7 p \\
6 s 6 p^{3} 7 s 7 p\end{array}$ & $\begin{array}{l}6 s^{2} 6 p^{4} \\
6 s^{2} 6 p^{3} 7 p \\
6 s^{2} 6 p^{3} 5 f \\
6 s^{2} 6 p^{3} 6 f \\
6 s^{2} 6 p^{2} 6 d^{2} \\
6 s^{2} 6 p^{2} 7 s^{2} \\
6 s^{2} 6 p^{2} 6 d 7 s \\
6 s^{2} 6 p^{2} 6 d 7 d \\
6 s^{2} 6 p^{2} 7 s 7 d \\
6 s^{2} 6 p^{2} 7 p 5 f \\
6 s^{2} 6 p^{2} 7 p 6 f \\
6 s^{2} 6 p 7 p^{3} \\
6 s^{2} 6 p 6 d^{2} 7 p \\
6 s^{2} 6 p 7 s^{2} 7 p \\
6 s^{2} 6 p 6 d 7 s 7 p \\
6 s 6 p^{4} 6 d \\
6 s 6 p^{4} 7 s \\
6 s 6 p^{3} 6 d 7 p \\
6 s 6 p^{3} 7 s 7 p \\
6 p^{6} \\
6 p^{5} 7 p \\
6 p^{4} 6 d^{2} \\
6 p^{4} 7 s^{2} \\
6 p^{4} 7 p^{2} \\
6 p^{4} 6 d 7 s\end{array}$ & $\begin{array}{l}6 s^{2} 6 p^{4} \\
6 s^{2} 6 p^{3} 7 p \\
6 s^{2} 6 p^{3} 5 f \\
6 s^{2} 6 p^{3} 6 f \\
6 s^{2} 6 p^{2} 6 d^{2} \\
6 s^{2} 6 p^{2} 7 s^{2} \\
6 s^{2} 6 p^{2} 6 d 7 s \\
6 s^{2} 6 p^{2} 6 d 7 d \\
6 s^{2} 6 p^{2} 7 s 7 d \\
6 s^{2} 6 p^{2} 7 p 5 f \\
6 s^{2} 6 p^{2} 7 p 6 f \\
6 s^{2} 6 p 7 p^{3} \\
6 s^{2} 6 p 6 d^{2} 7 p \\
6 s^{2} 6 p 7 s^{2} 7 p \\
6 s^{2} 6 p 6 d 7 s 7 p \\
6 s^{2} 6 p^{4} 6 d \\
6 s^{4} 7 s \\
6 p^{4} 6 p^{3} 6 d 7 p \\
6 s 6 p^{3} 7 s 7 p \\
6 p^{6} \\
6 p^{5} 7 p \\
6 p^{4} 6 d^{2} \\
6 p^{4} 7 s^{2} \\
6 p^{4} 7 p^{2} \\
6 p^{4} 6 d 7 s\end{array}$ \\
\hline Odd configurations & $\begin{array}{l}6 s^{2} 6 p^{3} 7 s \\
6 s^{2} 6 p^{3} 6 d \\
6 s^{2} 6 p^{3} 7 d\end{array}$ & $\begin{array}{l}6 s^{2} 6 p^{3} 7 s \\
6 s^{2} 6 p^{3} 6 d \\
6 s^{2} 6 p^{3} 7 d \\
6 s^{2} 6 p^{2} 6 d 7 p \\
6 s^{2} 6 p^{2} 7 s 7 p \\
6 s^{2} 6 p^{2} 6 d 5 f \\
6 s^{2} 6 p^{2} 6 d 6 f \\
6 s^{2} 6 p^{2} 7 s 5 f \\
6 s^{2} 6 p^{2} 7 s 6 f\end{array}$ & $\begin{array}{l}6 s^{2} 6 p^{3} 7 s \\
6 s^{2} 6 p^{3} 6 d \\
6 s^{2} 6 p^{3} 7 d \\
6 s^{2} 6 p^{2} 6 d 7 p \\
6 s^{2} 6 p^{2} 7 s 7 p \\
6 s^{2} 6 p^{2} 6 d 5 f \\
6 s^{2} 6 p^{2} 6 d 6 f \\
6 s^{2} 6 p^{2} 7 s 5 f \\
6 s^{2} 6 p^{2} 7 s 6 f \\
6 s^{2} 6 p 6 d^{3} \\
6 s^{2} 6 p 6 d^{2} 7 s \\
6 s^{2} 6 p 6 d 7 s^{2} \\
6 s^{2} 6 p 6 d 7 p^{2} \\
6 s^{2} 6 p 7 s 7 p^{2}\end{array}$ & $\begin{array}{l}6 s^{2} 6 p^{3} 7 s \\
6 s^{2} 6 p^{3} 6 d \\
6 s^{2} 6 p^{3} 7 d \\
6 s^{2} 6 p^{2} 6 d 7 p \\
6 s^{2} 6 p^{2} 7 s 7 p \\
6 s^{2} 6 p^{2} 6 d 5 f \\
6 s^{2} 6 p^{2} 6 d 6 f \\
6 s^{2} 6 p^{2} 7 s 5 f \\
6 s^{2} 6 p^{2} 7 s 6 f \\
6 s^{2} 6 p 6 d^{3} \\
6 s^{2} 6 p 6 d^{2} 7 s \\
6 s^{2} 6 p 6 d 7 s^{2} \\
6 s^{2} 6 p 6 d 7 p^{2} \\
6 s^{2} 6 p 7 s 7 p^{2} \\
6 s^{2} 6 p^{5} \\
6 s^{4} p^{4} 7 p \\
6 s 6 p^{3} 6 d^{2} \\
6 s 6 p^{3} 7 s^{2} \\
6 s 6 p^{3} 7 p^{2} \\
6 s 6 p^{3} 6 d 7 s\end{array}$ & $\begin{array}{l}6 s^{2} 6 p^{3} 7 s \\
6 s^{2} 6 p^{3} 6 d \\
6 s^{2} 6 p^{3} 7 d \\
6 s^{2} 6 p^{2} 6 d 7 p \\
6 s^{2} 6 p^{2} 7 s 7 p \\
6 s^{2} 6 p^{2} 6 d 5 f \\
6 s^{2} 6 p^{2} 6 d 6 f \\
6 s^{2} 6 p^{2} 7 s 5 f \\
6 s^{2} 6 p^{2} 7 s 6 f \\
6 s^{2} 6 p 6 d^{3} \\
6 s^{2} 6 p 6 d^{2} 7 s \\
6 s^{2} 6 p 6 d 7 s^{2} \\
6 s^{2} 6 p 6 d 7 p^{2} \\
6 s^{2} 6 p 7 s 7 p^{2} \\
6 s^{5} p^{5} \\
6 s^{4} 7 p^{4} 7 p \\
6 s^{3} 6 p^{2} \\
6 s 6 p^{3} 7 s^{2} \\
6 s 6 p^{3} 7 p^{2} \\
6 s 6 p^{3} 6 d 7 s \\
6 p^{5} 6 d \\
6 p^{5} 7 s \\
6 p^{4} 6 d 7 p \\
6 p^{4} 7 s 7 p\end{array}$ & $\begin{array}{l}6 s^{2} 6 p^{3} 7 s \\
6 s^{2} 6 p^{3} 6 d \\
6 s^{2} 6 p^{3} 7 d \\
6 s^{2} 6 p^{2} 6 d 7 p \\
6 s^{2} 6 p^{2} 7 s 7 p \\
6 s^{2} 6 p^{2} 6 d 5 f \\
6 s^{2} 6 p^{2} 6 d 6 f \\
6 s^{2} 6 p^{2} 7 s 5 f \\
6 s^{2} 6 p^{2} 7 s 6 f \\
6 s^{2} 6 p 6 d^{3} \\
6 s^{2} 6 p 6 d^{2} 7 s \\
6 s^{2} 6 p 6 d 7 s^{2} \\
6 s^{2} 6 p 6 d 7 p^{2} \\
6 s^{2} 6 p 7 s 7 p^{2} \\
6 s^{5} p^{5} \\
6 s^{4} 7 p \\
6 s^{4} 6 p^{3} 6 d^{2} \\
6 s 6 p^{3} 7 s^{2} \\
6 s 6 p^{3} 7 p^{2} \\
6 s 6 p^{3} 6 d 7 s \\
6 p^{5} 6 d \\
6 p^{5} 7 s \\
6 p^{4} 6 d 7 p \\
6 p^{4} 7 s 7 p\end{array}$ \\
\hline Core-polarization parameters & & & & & & $\begin{array}{l}\alpha_{d}=2.00 a_{0}^{3} \\
r_{c}=1.17 a_{0} \\
\text { (Po VII core) }\end{array}$ \\
\hline
\end{tabular}

In each of these approaches (from $A$ to $F$ ), the final wavefunctions were obtained by a parametric fit of the calculated energy levels to the experimental values taken from $[20,21]$. More precisely, for the even parity, 8 level energies were used to optimize the average energy, $E_{a v}$, the Slater electrostic interaction integral, $F^{2}(6 \mathrm{p}, 6 \mathrm{p})$, the spinorbit parameter, $\zeta_{6 \mathrm{p}}$, and the effective interaction parameter, $\alpha$, in the $6 \mathrm{p}^{4}$ configuration as well as the average energy and the spin-orbit parameter, $\zeta_{7 \mathrm{p}}$, in the $6 \mathrm{p}^{3} 7 \mathrm{p}$ configuration. In the case of odd parity, 9 experimental energy levels were used to adjust the numerical values of the average energy, $E_{a v}$, the Slater direct, $F^{2}(6 \mathrm{p}, 6 \mathrm{p})$, and exchange, $G^{1}(6 \mathrm{p}, 7 \mathrm{~s})$, integrals in $6 \mathrm{p}^{3} 7 \mathrm{~s}$ configuration, together with the average energy, $E_{a v}$, and the Slater exchange integrals, $G^{1}(6 \mathrm{p}, 6 \mathrm{~d})$ and $G^{3}(6 \mathrm{p}, 6 \mathrm{~d})$, in $6 \mathrm{p}^{3} 6 \mathrm{~d}$ configuration, with the constraint that the ratio between these two latter parameters is kept fixed during the fitting process. Note also that the $6 p^{3} 7 d$ average energy was adjusted using the experimental value of $6 \mathrm{p}^{3} 7 \mathrm{~d}(\mathrm{~J}=2)$ level at $59469.66 \mathrm{~cm}^{-1}$ [21]. In addition, the ab initio HFR values for all the other Slater integrals, $F^{k}, G^{k}$ and $R^{k}$, not adjusted semi-empirically were scaled down by a 
factor 0.80, as suggested by Cowan [24]. In Table 2, the lowest energy levels obtained below $59,500 \mathrm{~cm}^{-1}$ using the most sophisticated model (F) are presented and compared with available experimental values. The main eigenvector components are also given for each level in the same table. In the work published by Charles [21], many levels were not identified by a full spectroscopic designation, some of them being even listed with an

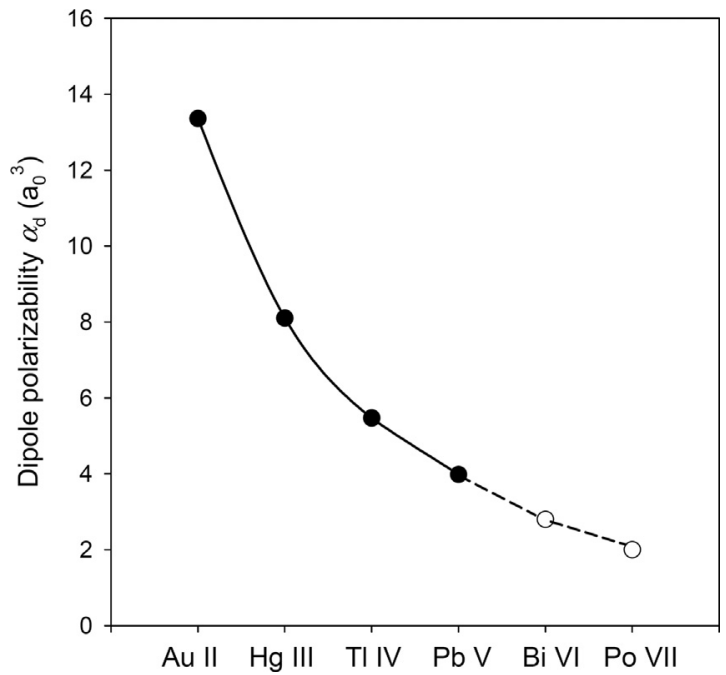

Fig. 1. Dipole polarizability along the platinum isoelectronic sequence. Full circles are theoretical values taken from [28]. Open circles connected with a dotted line represent extrapolated values. uncertain value of the $J$-quantun number. These gaps are now filled for the lowest excited levels of $6 p^{4}, 6 p^{3} 7 p$ $6 \mathrm{p}^{3} 6 \mathrm{~d}, 6 \mathrm{p}^{3} 7 \mathrm{~s}$ and $6 \mathrm{p}^{3} 7 \mathrm{~d}$ configurations reported in Table 2 . In particular, the three even-parity levels at 50,681.28, $50,934.89$ and $51,636.42 \mathrm{~cm}^{-1}$ were clearly identified in our calculations as belonging to the $6 \mathrm{p}^{3}\left({ }^{4} \mathrm{~S}\right) 7 \mathrm{p}{ }^{5} \mathrm{P}$ multiplet with $J=1,2$ and 3, respectively, which seems to contradict the assumptions made by Charles [21] who suggested the values $J=3$ ?, $J=$ ? and $J=1$ or 2 for the same levels, respectively. However, it is worth noting that the spectroscopic designations obtained in the present work are confirmed by the same order of fine-structure levels observed within the $5 \mathrm{p}^{3}\left({ }^{4} \mathrm{~S}\right) 6 \mathrm{p}{ }^{5} \mathrm{P}$ multiplet in the homolog Te I spectrum $[20,29]$. In the odd parity, the Po I levels at $54,250.26,55,923.80$ and $57,078.05 \mathrm{~cm}^{-1}$, not clearly identified by Charles [21] are now attributed to $6 \mathrm{p}^{3}\left({ }^{4} \mathrm{~S}\right) 6 \mathrm{~d}$ ${ }^{3} D_{1}, 6 p^{3}\left({ }^{2} D\right) 7 s^{3} D_{2}$ and $6 p^{3}\left({ }^{2} D\right) 7 s^{3} D_{1}$, respectively, the two latter states being however very strongly mixed with $6 p^{3}\left({ }^{4} S\right) 7 s{ }^{5} S_{2}$ and $6 p^{3}\left({ }^{4} S\right) 7 s^{3} S_{1}$.

\section{Radiative parameters}

The theoretical oscillator strengths, log gf, computed in the present work using the HFR models A-F described above are compared in Table 3 for 31 Po I lines involving all the experimentally known levels listed in Table 2. Note that transitions affected by severe cancellation effects in the calculation of the line strength, i.e. transitions for which the cancellation factor $\mathrm{CF}$ as defined by Cowan [24] is smaller than 0.01, were removed from the table.

Table 2

Lowly excited energy levels (below $59,500 \mathrm{~cm}^{-1}$ ) in $6 \mathrm{p}^{4}, 6 \mathrm{p}^{3} 6 \mathrm{~d}, 6 \mathrm{p}^{3} 7 \mathrm{~s}, 6 \mathrm{p}^{3} 7 \mathrm{p}$ and $6 \mathrm{p}^{3} 7 \mathrm{~d}$ configurations of Po I.

\begin{tabular}{|c|c|c|c|c|c|c|}
\hline$E_{\exp }\left(\mathrm{cm}^{-1}\right)^{\mathrm{a}}$ & $E_{\text {calc }}\left(\mathrm{cm}^{-1}\right)^{\mathrm{b}}$ & $\Delta E\left(\mathrm{~cm}^{-1}\right)$ & $J$ & 1st Component & 2nd Component & 3rd Component \\
\hline \multicolumn{7}{|l|}{ Even parity } \\
\hline 0.00 & 0 & 0 & 2 & $78 \% 6 p^{4}{ }^{3} \mathrm{P}$ & $20 \% 6 p^{41} D$ & \\
\hline 7514.69 & 7516 & -1 & 0 & $55 \% 6 \mathrm{p}^{4}{ }^{3} \mathrm{P}$ & $43 \% 6 p^{4}{ }^{1} s$ & \\
\hline 16831.61 & 16833 & -1 & 1 & $98 \% 6 p^{43} \mathrm{P}$ & & \\
\hline 21679.11 & 21680 & -1 & 2 & $78 \% 6 \mathrm{p}^{4}{ }^{1} \mathrm{D}$ & $20 \% 6 p^{4}{ }^{3} \mathrm{P}$ & \\
\hline 42718.00 & 42717 & 1 & 0 & $54 \% 6 \mathrm{p}^{4}{ }^{1} \mathrm{~S}$ & $43 \% 6 \mathrm{p}^{4}{ }^{3} \mathrm{P}$ & \\
\hline 50681.28 & 50773 & -92 & 1 & $34 \% 6 p^{3}\left({ }^{4} S\right) 7 p^{5} \mathrm{P}$ & $16 \% 6 p^{3}\left({ }^{4} S\right) 7 p{ }^{3} P$ & $15 \% 6 p^{3}\left({ }^{2} \mathrm{P}\right) 7 \mathrm{p}^{3} \mathrm{P}$ \\
\hline 50934.89 & 50838 & 97 & 2 & $40 \% 6 p^{3}\left({ }^{4} S\right) 7 p{ }^{5} \mathrm{P}$ & $15 \% 6 p^{3}\left({ }^{2} P\right) 7 p^{3} D$ & $13 \% 6 \mathrm{p}^{3}\left({ }^{2} \mathrm{P}\right) 7 \mathrm{p}{ }^{3} \mathrm{P}$ \\
\hline \multirow[t]{4}{*}{51636.42} & 51641 & -5 & 3 & $52 \% 6 \mathrm{p}^{3}\left({ }^{4} \mathrm{~s}\right) 7 \mathrm{p}{ }^{5} \mathrm{P}$ & $31 \% 6 p^{3}\left({ }^{2} P\right) 7 p^{3} D$ & $8 \% 6 p^{3}\left({ }^{2} D\right) 7 p^{3} F$ \\
\hline & 51896 & & 1 & $31 \% 6 p^{3}\left({ }^{4} S\right) 7 p{ }^{3} P$ & $19 \% 6 p^{3}\left({ }^{4} S\right) 7 p{ }^{5} P$ & $16 \% 6 p^{3}\left({ }^{2} \mathrm{P}\right) 7 \mathrm{p}^{3} \mathrm{~s}$ \\
\hline & 52853 & & 2 & $38 \% 6 p^{3}\left({ }^{4} S\right) 7 p^{3} P$ & $17 \% 6 \mathrm{p}^{3}\left({ }^{2} \mathrm{P}\right) 7 \mathrm{p}{ }^{1} \mathrm{D}$ & $13 \% 6 p^{3}\left({ }^{2} \mathrm{P}\right) 7 \mathrm{p}^{3} \mathrm{P}$ \\
\hline & 53847 & & 0 & $55 \% 6 \mathrm{p}^{3}\left({ }^{4} \mathrm{~S}\right) 7 \mathrm{p}{ }^{3} \mathrm{P}$ & $19 \% 6 \mathrm{p}^{3}\left({ }^{2} \mathrm{P}\right) 7 \mathrm{p}{ }^{1} \mathrm{~S}$ & $12 \% 6 p^{3}\left({ }^{2} D\right) 7 p^{3} P$ \\
\hline \multicolumn{7}{|l|}{ Odd parity } \\
\hline 39081.19 & 38837 & 244 & 2 & $52 \% 6 \mathrm{p}^{3}\left({ }^{4} \mathrm{~S}\right) 7 \mathrm{~s}{ }^{5} \mathrm{~s}$ & $32 \% 6 \mathrm{p}^{3}\left({ }^{2} \mathrm{P}\right) 7 \mathrm{~s}^{3} \mathrm{P}$ & $9 \% 6 \mathrm{p}^{3}\left({ }^{2} \mathrm{D}\right) 7 \mathrm{~s}^{3} \mathrm{D}$ \\
\hline 40802.70 & 40551 & 252 & 1 & $43 \% 6 p^{3}\left({ }^{4} s\right) 7 s{ }^{3} s$ & $22 \% 6 \mathrm{p}^{3}\left({ }^{2} \mathrm{P}\right) 7 \mathrm{~s}{ }^{1} \mathrm{P}$ & $20 \% 6 \mathrm{p}^{3}\left({ }^{2} \mathrm{D}\right) 7 \mathrm{~s}^{3} \mathrm{D}$ \\
\hline 51713.09 & 51846 & -133 & 2 & $28 \% 6 p^{3}\left({ }^{4} S\right) 6 d{ }^{5} D$ & $18 \% 6 p^{3}\left({ }^{4} s\right) 6 d^{3} D$ & $15 \% 6 p^{3}\left({ }^{2} P\right) 6 d^{3} D$ \\
\hline 52098.93 & 52213 & -114 & 3 & $47 \% 6 \mathrm{p}^{3}\left({ }^{4} \mathrm{~s}\right) 6 \mathrm{~d}{ }^{5} \mathrm{D}$ & $17 \% 6 \mathrm{p}^{3}\left({ }^{2} \mathrm{P}\right) 6 \mathrm{~d}^{3} \mathrm{D}$ & $14 \% 6 \mathrm{p}^{3}\left({ }^{2} \mathrm{P}\right) 6 \mathrm{~d}^{3} \mathrm{~F}$ \\
\hline \multirow[t]{2}{*}{52532.12} & 52375 & 157 & 1 & $44 \% 6 p^{3}\left({ }^{4} s\right) 6 d^{5} D$ & $17 \% 6 \mathrm{p}^{3}\left({ }^{2} \mathrm{P}\right) 6 \mathrm{~d}^{3} \mathrm{P}$ & $12 \% 6 \mathrm{p}^{3}\left({ }^{2} \mathrm{P}\right) 6 \mathrm{~d}^{3} \mathrm{D}$ \\
\hline & 52835 & & 4 & $53 \% 6 p^{3}\left({ }^{4} S\right) 6 d{ }^{5} D$ & $31 \% 6 p^{3}\left({ }^{2} P\right) 6 d^{3} F$ & $8 \% 6 p^{3}\left({ }^{2} D\right) 6 d^{3} G$ \\
\hline \multirow[t]{3}{*}{53027.61} & 52863 & 165 & 2 & $26 \% 6 p^{3}\left({ }^{4} S\right) 6 d{ }^{3} D$ & $21 \% 6 p^{3}\left({ }^{4} S\right) 6 d{ }^{5} D$ & $6 \% 6 p^{3}\left({ }^{2} D\right) 6 d{ }^{3} P$ \\
\hline & 52904 & & 0 & $54 \% 6 \mathrm{p}^{3}\left({ }^{4} s\right) 6 \mathrm{~d}^{5} \mathrm{D}$ & $30 \% 6 \mathrm{p}^{3}\left({ }^{2} \mathrm{P}\right) 6 \mathrm{~d}{ }^{3} \mathrm{P}$ & $7 \% 6 \mathrm{p}^{3}\left({ }^{2} \mathrm{D}\right) 6 \mathrm{~d}^{3} \mathrm{P}$ \\
\hline & 54000 & & 3 & $46 \% 6 p^{3}\left({ }^{4} S\right) 6 d^{3} D$ & $20 \% 6 \mathrm{p}^{3}\left({ }^{2} \mathrm{P}\right) 6 \mathrm{~d}{ }^{1} \mathrm{~F}$ & $11 \% 6 p^{3}\left({ }^{2} D\right) 6 d^{3} G$ \\
\hline 54250.26 & 54316 & -65 & 1 & $44 \% 6 \mathrm{p}^{3}\left({ }^{4} S\right) 6 \mathrm{~d}^{3} \mathrm{D}$ & $16 \% 6 \mathrm{p}^{3}\left({ }^{2} \mathrm{P}\right) 6 \mathrm{~d}^{1} \mathrm{P}$ & $10 \% 6 \mathrm{p}^{3}\left({ }^{2} \mathrm{P}\right) 6 \mathrm{~d}^{3} \mathrm{P}$ \\
\hline 55923.80 & 56107 & -183 & 2 & $37 \% 6 \mathrm{p}^{3}\left({ }^{2} \mathrm{D}\right) 7 \mathrm{~s}^{3} \mathrm{D}$ & $36 \% 6 p^{3}\left({ }^{4} S\right) 7 s{ }^{5} S$ & $16 \% 6 \mathrm{p}^{3}\left({ }^{2} \mathrm{D}\right) 7 \mathrm{~s}{ }^{1} \mathrm{D}$ \\
\hline 57078.05 & 57321 & -243 & 1 & $46 \% 6 \mathrm{p}^{3}\left({ }^{2} \mathrm{D}\right) 7 \mathrm{~s}^{3} \mathrm{D}$ & $36 \% 6 p^{3}\left({ }^{4} s\right) 7 s^{3} s$ & $3 \% 6 p^{3}\left({ }^{4} S\right) 7 d^{3} D$ \\
\hline 59469.66 & 59456 & 14 & 2 & $30 \% 6 p^{3}\left({ }^{4} S\right) 7 d^{5} D$ & $20 \% 6 p^{3}\left({ }^{4} S\right) 7 d^{3} D$ & $14 \% 6 p^{3}\left({ }^{2} P\right) 7 d^{3} D$ \\
\hline
\end{tabular}

a Experimental energy levels from [21].

${ }^{b}$ Calculated energy levels as obtained in the present work using the HFR(F) model. 
Table 3

Calculated oscillator strengths (log gf) for transitions in Po I using the different HFR models considered in the present work.

\begin{tabular}{|c|c|c|c|c|c|c|c|c|c|c|}
\hline \multirow{2}{*}{$\frac{\lambda(\mathrm{nm})^{\mathrm{a}}}{175.199 *}$} & \multicolumn{2}{|c|}{ Lower level $\left(\mathrm{cm}^{-1}\right)^{\mathrm{b}}$} & \multicolumn{2}{|c|}{ Upper level $\left(\mathrm{cm}^{-1}\right)^{\mathrm{b}}$} & \multirow{2}{*}{$\begin{array}{l}\text { A } \\
-1.02\end{array}$} & \multirow{2}{*}{$\begin{array}{l}\text { B } \\
-0.96\end{array}$} & \multirow{2}{*}{$\begin{array}{l}\text { C } \\
-1.02\end{array}$} & \multirow{2}{*}{$\begin{array}{l}\text { D } \\
-0.93\end{array}$} & \multirow{2}{*}{$\begin{array}{l}\text { E } \\
-0.87\end{array}$} & \multirow{2}{*}{$\begin{array}{l}F \\
-0.85\end{array}$} \\
\hline & 0.00 & $J=2$ (even) & 57078.05 & $J=1($ odd $)$ & & & & & & \\
\hline $178.815 *$ & 0.00 & $J=2$ (even) & 55923.80 & $J=2($ odd $)$ & -0.29 & -0.30 & -0.29 & -0.38 & -0.38 & -0.36 \\
\hline $184.331 *$ & 0.00 & $J=2$ (even) & 54250.26 & $J=1$ (odd) & -0.78 & -0.85 & -0.84 & -0.78 & -0.79 & -0.81 \\
\hline $188.581 *$ & 0.00 & $J=2$ (even) & 53027.61 & $J=2($ odd $)$ & -0.05 & -0.11 & -0.10 & -0.15 & -0.14 & -0.15 \\
\hline $190.360 *$ & 0.00 & $J=2$ (even) & 52532.12 & $J=1($ odd $)$ & -1.61 & -1.66 & -1.61 & -2.04 & -1.96 & -1.98 \\
\hline $191.943 *$ & 0.00 & $J=2$ (even) & 52098.93 & $J=3$ (odd) & -0.63 & -0.76 & -0.68 & -0.63 & -0.60 & -0.61 \\
\hline $201.697 *$ & 7514.69 & $J=0$ (even) & 57078.05 & $J=1($ odd $)$ & -0.98 & -1.07 & -1.00 & -1.08 & -1.18 & -1.19 \\
\hline 213.9024 & 7514.69 & $J=0$ (even) & 54250.26 & $J=1($ odd $)$ & -0.21 & -0.25 & -0.25 & -0.25 & -0.24 & -0.26 \\
\hline 222.0670 & 7514.69 & $J=0$ (even) & 52532.12 & $J=1($ odd $)$ & -1.21 & -1.27 & -1.31 & -1.27 & -1.25 & -1.26 \\
\hline 234.4606 & 16831.61 & $J=1$ (even) & 59469.66 & $J=2($ odd $)$ & -1.95 & -1.84 & -1.88 & -1.78 & -1.89 & -1.87 \\
\hline 245.0076 & 0.00 & $J=2$ (even) & 40802.70 & $J=1$ (odd) & -0.06 & -0.09 & -0.08 & -0.11 & -0.11 & -0.09 \\
\hline 248.3942 & 16831.61 & $J=1$ (even) & 57078.05 & $J=1($ odd $)$ & -0.23 & -0.23 & -0.24 & -0.23 & -0.23 & -0.21 \\
\hline $255.729 *$ & 16831.61 & $J=1$ (even) & 55923.80 & $J=2$ (odd) & -1.16 & -1.12 & -1.15 & -1.14 & -1.16 & -1.14 \\
\hline 255.8008 & 0.00 & $J=2$ (even) & 39081.19 & $J=2$ (odd) & -0.47 & -0.46 & -0.47 & -0.46 & -0.44 & -0.42 \\
\hline 264.5376 & 21679.11 & $J=2$ (even) & 59469.66 & $J=2$ (odd) & -1.56 & -1.51 & -1.58 & -1.46 & -1.56 & -1.54 \\
\hline 276.1920 & 16831.61 & $J=1$ (even) & 53027.61 & $J=2$ (odd) & -1.18 & -1.23 & -1.23 & -1.13 & -1.17 & -1.21 \\
\hline 280.0256 & 16831.61 & $J=1$ (even) & 52532.12 & $J=1$ (odd) & -1.87 & -1.96 & -1.89 & -2.05 & -2.02 & -1.96 \\
\hline 282.4112 & 21679.11 & $J=2$ (even) & 57078.05 & $J=1$ (odd) & -1.15 & -1.17 & -1.17 & -1.15 & -1.13 & -1.11 \\
\hline 286.6010 & 16831.61 & $J=1$ (even) & 51713.09 & $J=2($ odd $)$ & -1.54 & -1.56 & -1.63 & -1.72 & -1.78 & -1.80 \\
\hline 291.9306 & 21679.11 & $J=2$ (even) & 55923.80 & $J=2($ odd $)$ & -1.09 & -1.13 & -1.12 & -1.13 & -1.15 & -1.14 \\
\hline 300.3209 & 7514.69 & $J=0$ (even) & 40802.70 & $J=1$ (odd) & -0.81 & -0.82 & -0.82 & -0.81 & -0.82 & -0.80 \\
\hline 306.9310 & 21679.11 & $J=2$ (even) & 54250.26 & $J=1($ odd $)$ & -2.01 & -2.04 & -1.97 & -2.34 & -2.40 & -2.36 \\
\hline 324.0241 & 21679.11 & $J=2$ (even) & 52532.12 & $J=1$ (odd) & -1.67 & -1.72 & -1.71 & -1.88 & -1.90 & -1.85 \\
\hline 328.6384 & 21679.11 & $J=2$ (even) & 52098.93 & $J=3$ (odd) & -1.98 & -1.96 & -2.00 & -2.00 & -2.12 & -2.14 \\
\hline 417.0517 & 16831.61 & $J=1$ (even) & 40802.70 & $J=1$ (odd) & -1.30 & -1.40 & -1.35 & -1.42 & -1.49 & -1.49 \\
\hline 574.4848 & 21679.11 & $J=2$ (even) & 39081.19 & $J=2($ odd $)$ & -2.63 & -2.63 & -2.67 & -2.52 & -2.61 & -2.62 \\
\hline $696.184 *$ & 42718.00 & $J=0$ (even) & 57078.05 & $J=1$ (odd) & -2.53 & -2.57 & -2.52 & -2.60 & -2.46 & -2.46 \\
\hline 796.2618 & 39081.19 & $J=2$ (odd) & 51636.42 & $J=3$ (even) & 0.57 & 0.54 & 0.54 & 0.53 & 0.53 & 0.52 \\
\hline 843.3871 & 39081.19 & $J=2$ (odd) & 50934.89 & $J=2$ (even) & 0.32 & 0.27 & 0.28 & 0.27 & 0.28 & 0.27 \\
\hline 861.8255 & 39081.19 & $J=2($ odd $)$ & 50681.28 & $J=1$ (even) & 0.02 & -0.03 & -0.03 & -0.06 & -0.06 & -0.07 \\
\hline $986.683 *$ & 40802.70 & $J=1($ odd $)$ & 50934.89 & $J=2$ (even) & -0.40 & -0.41 & -0.41 & -0.46 & -0.43 & -0.45 \\
\hline
\end{tabular}

a Experimental wavelengths in vacuum (below $200 \mathrm{~nm}$ ) and in air (above $200 \mathrm{~nm}$ ) taken from [21]. Values with an asterisk * were deduced from the experimental energy levels.

${ }^{\mathrm{b}}$ Experimental energy level values taken from [21].

It is indeed well-known that the computed transition rates for such (usually weak) transitions may be expected to show very large errors. For information, these doubtful transition rates concern spectral lines situated at 168.153 , 193.375, 267.167, 318.902, 332.860, 449.321, 522.769 and $866.895 \mathrm{~nm}$.

The influence of the different types of electron correlation on oscillator strength calculations in polonium atom is illustrated in Fig. 2. In this figure, the ratios between $g f$ values computed using our different HFR models are compared for all the Po I lines listed in Table 3, numbered according to the order of appearance in that table. When looking at Fig. 2, one can observe that single and double excitations from both the $6 \mathrm{~s}$ and $6 \mathrm{p}$ subshells (models BE) play a non negligible role in the overall calculation of transition rates of interest while core-polarization effects from the Po VII $\left[1 \mathrm{~s}^{2} \ldots 5 \mathrm{~d}^{10}\right]$ ionic core (model $\mathrm{F}$ ) are rather small. This can be illustrated by the mean ratios of oscillator strengths obtained with two successive models for which the values were found to be equal to $g f(\mathrm{~B}) / g f$ $(\mathrm{A})=0.94 \pm 0.11, g f(C) / g f(B)=1.01 \pm 0.09, g f(D) / g f(C)=0.96$ $\pm 0.23, \quad g f(\mathrm{E}) / g f(\mathrm{D})=0.98 \pm 0.13 \quad$ and $\quad g f(\mathrm{~F}) / g f(\mathrm{E})=1.01$ \pm 0.05 where the uncertainty represents the standard deviation.

However, when looking into more details, we note that the most important electron correlation effects on the oscillator strengths depend on the type of transition considered. As an example, for the $6 p^{4}-6 p^{3} 6 d$ transitions, the most important changes in the $g f$-values are observed when considering models B and D. This is essentially because the $6 s^{2} 6 p^{2} 6 d^{2}$ and the $6 s 6 p^{4} 6 d$ configurations included in the $6 s^{2} 6 p^{4}$ initial state in models $B$ and $D$, respectively, have allowed dipole transitions to the $6 s^{2} 6 p^{3} 6 d$ configuration. Furthermore, the radial dipole integral $6 d \rightarrow 6 p$ involved in the transition from the $6 s^{2} 6 p^{2} 6 d^{2}$ configuration has the same magnitude as the dipole matrix elements which connect the $6 \mathrm{p}^{4}$ and $6 \mathrm{p}^{3} 6 \mathrm{~d}$ main configurations. On the other hand, the equivalent configuration $\left(6 \mathrm{~s}^{2} 6 \mathrm{p} 6 \mathrm{~d}^{3}\right)$ included in the $6 s^{2} 6 \mathrm{p}^{3} 6 \mathrm{~d}$ final state in model $\mathrm{C}$ does not have an allowed transition to the $6 s^{2} 6 \mathrm{p}^{4}$ initial state which explains why it is more important to include the $6 \mathrm{p}^{2} \rightarrow 6 \mathrm{~d}^{2}$ double excitation in the initial than in the final state. Also the $6 s \rightarrow 6 d$ core excitation included in model D leads to a configuration $\left(6 s 6 p^{4} 6 \mathrm{~d}\right)$ with an allowed dipole transition to the $6 s^{2} 6 p^{3} 6 d$ final state which is not the case for the $6 s^{2} \rightarrow 6 d^{2}$ double excitation considered in model E. A similar discussion can be done concerning the influence of $6 \mathrm{p}^{2} \rightarrow$ $7 s^{2}$ and $6 s \rightarrow 7 s$ core excitations on the oscillator strengths corresponding to the $6 \mathrm{p}^{4}-6 \mathrm{p}^{3} 7 \mathrm{~s}$ transitions. The situation is different for the $6 p^{3} 7 s-6 p^{3} 7 p$ transitions (i.e. the four last transitions in Table 3 and in Fig. 2). In this case, the computed oscillator strengths are only slightly sensitive 


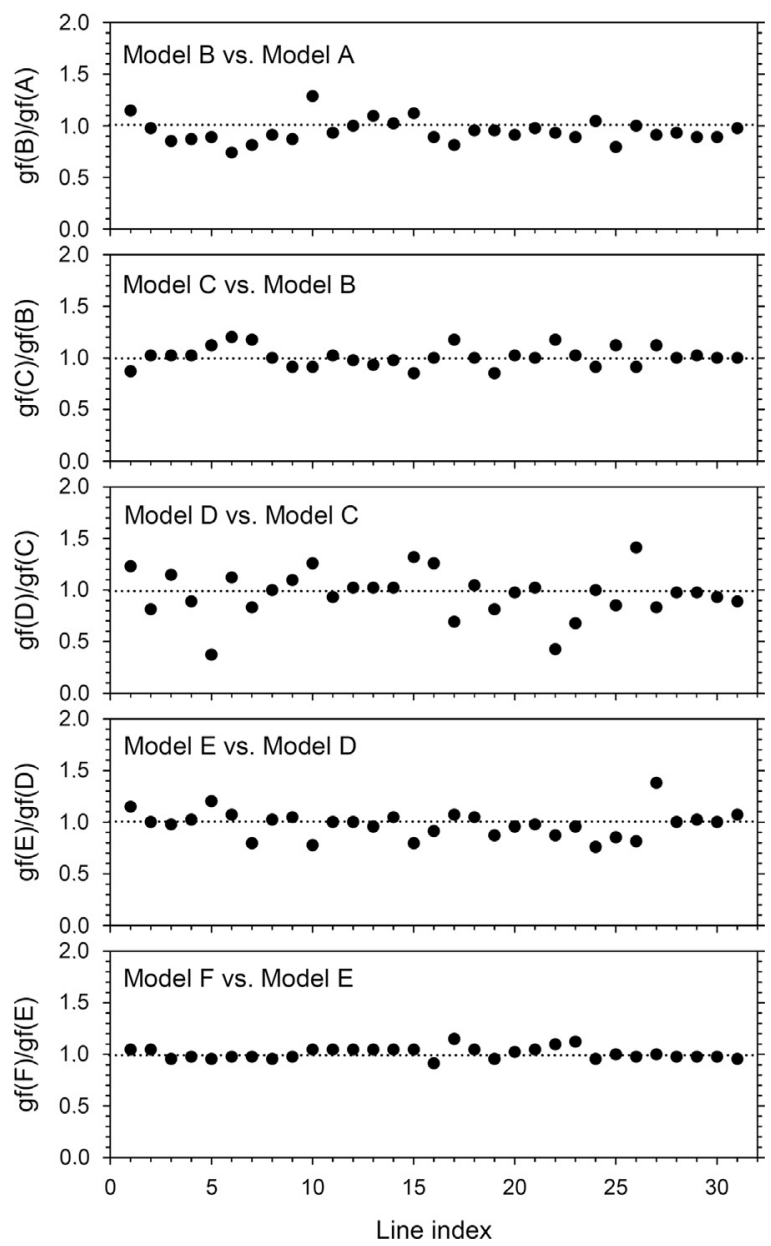

Fig. 2. Comparison between oscillator strengths obtained in the present work using different HFR models. In each panel, the $y$-axis gives the ratio of $g$-values computed with two successive models, i.e. including an increasing number of interacting configurations (see text). The $x$-axis of the plots corresponds to the Po I spectral line indexes, which are assigned according to the order of appearance in Table 3 (i.e. by increasing wavelengths).

(within 10\%) to single excitations from the $6 \mathrm{p}$ (model B) and from the 6s (model D) core subshells while double excitations from the same subshells have a negligible effect.

\section{Conclusion}

Different physical models based on the pseudorelativistic Hartree-Fock method have been used for modeling the atomic structure and for computing radiative parameters in neutral polonium. They have emphasized the dominating influence of core-valence and core-core correlation for the $6 p^{4}-6 p^{3} 6 d$ and $6 p^{4}-6 p^{3} 7 s$ transitions. They also allowed us to provide a new set of decay rates for lines involving the lowest states in the $6 \mathrm{p}^{4}, 6 \mathrm{p}^{3} 6 \mathrm{~d}$, $6 p^{3} 7 s, 6 p^{3} 7 p$ and $6 p^{3} 7 d$ configurations and to give a reliable spectroscopic designation to some energy levels not clearly classified in previous works. This investigation is expected to provide a theoretical support to laserspectroscopy experiments at ISOLDE and to check other theoretical methods that will be used to compute hyperfine structure and isotope shift electronic parameters in the future. In addition, the new set of oscillator strengths obtained in the present work provides the basic information for a quantitative analysis of the polonium content in some chemically peculiar stars.

\section{Acknowledgments}

This work was performed thanks to the support of the Belgian Research Initiative on eXotic nuclei for atomic, nuclear and astrophysics studies - BRIX (BELSPO IAP-VII Network no. P7/12). The author is Research Director of the F.R.S.-FNRS (Grant no. FC 49142). Financial support from this organization is also greatly acknowledged.

\section{References}

[1] Fry C, Thoennessen M. Discovery of the thallium, lead, bismuth and polonium isotopes. At Data Nucl Data Tables 2013;99:365-89.

[2] Kowalewska D, Bekk K, Göring S, Hanser A, Kälber W, Meisel G, et al. Isotope shifts and hyperfine structure in polonium isotopes by atomic-beam laser spectroscopy. Phys Rev A 1991;44:R1442-5.

[3] Cocolios TE, Marsh BA, Fedoseev VN, Franchoo S, Huber G, Huyse M et al. Resonant laser ionization of polonium at RILIS-ISOLDE for the study of ground- and isomer-state properties. Nucl Instrum Math Phys Res B 2008;266:4403-6.

[4] Cocolios TE, Dexters W, Seliverstov MD, Andreyev AN, Antalic S, Barzakh AE, et al. Early onset of ground state deformation in neutron deficient polonium isotopes. Phys Rev Lett 2011;106: 052503.

[5] Fritzsche S. Large-scale accurate structure calculations for open-shell atoms and ions. Phys Scr T 2002;100:37-46.

[6] Cocolios TE, Andreyev AN, Antalic S, Barzakh AE, Bastin B, Büscher J, et al. Early onset of deformation in the neutron-deficient polonium isotopes. J Phys Conf Ser 2012;381:012072.

[7] Cowley CR, Bidelman WP, Hubrig S, Mathys G, Bord DJ. On the possible presence of promethium in the spectra of HD 101065 (Przybylski's star) and HD 965. Astron Astrophys 2004;419:1087-93.

[8] Gopka VF, Yushchenko AV, Shavrina AV, Mkrtichian DE, Hatzes AP, Andrievsky SM, et al. On the radioactive shells in peculiar main sequence stars: the phenomenon of Przybylski's star. In: Zverko J, Ziznovsky J, Adelman SJ, Weiss WW, editors. Proceedings of IAU symposium. The A-star puzzle, vol. 224; 2004. p. 734-42.

[9] Bidelman WP. Tc and other unstable elements in Przybylski's star. In: Barnes TG, Bash FN, editors. Proceedings of cosmic abundances as records of stellar evolution and nucleosynthesis. ASP Conference Series, vol. 336; 2005. p. 309.

[10] Gopka VF, Yushchenko AV, Goriely S Shavrina AV, Kang YW. Radioactive elements in stellar atmospheres. In: Proceedings of the international symposium on origin of matter and evolution of galaxies: new horizon of nuclear astrophysics and cosmology. AIP Conference Proceedings, vol. 847; 2006. p. 389.

[11] Goriely S, Arnould M. Actinides: how well do we know their stellar production? Astron Astrophys 2001;379:1113.

[12] Gopka VF, Yushchenko AV, Shavrina AV, Mkrtichian DE, Hatzes AP, Andrievsky SM, et al. About the possibility of explanation of the spectrum of Przybylski's star by the lines of radioactive elements. Proceedings of the international symposium on nuclear astrophysics - nuclei in the cosmos - IX. Geneva, Switzerland: CERN; 2006.

[13] Yushchenko AV, Gopka V, Goriely S, Lambert D, Shavrina A, Kang YW, et al. Interplay between diffusion, accretion and nuclear reactions in the atmospheres of Sirius and Przybylski's star. In: Kang YW, Lee HW, Cheng KS, Leung KC, editors. The seventh Pacific RIM conference on stellar astrophysics. ASP Conference Series, vol. 362; 2007. p. 46-53.

[14] Goriely S. Nucleosynthesis by accelerated particles to account for the surface composition of HD 101065. Astron Astrophys 2007;466:619.

[15] Palmeri P, Quinet P, Biémont E, Yushchenko AV, Jorissen A, Van Eck S. Radiative decay of the $4 d^{5}\left({ }^{6} S\right) 5 p z^{5,7} P^{\circ}$ states in Tc II: comparsion along the homologous and isoelectronic sequences. Application to astrophysics. Mon Not R Astron Soc 2007;374:63-71. 
[16] Fivet V, Quinet P, Biémont E, Jorissen A, Yushchenko AV, Van Eck S. Transition probabilities in singly ionized promethium and the identification of Pm II lines in Przybylski's star and HR 465. Mon Not R Astron Soc 2007;380:771-80.

[17] Biémont E, Quinet P, Van Renterghem V. Theoretical investigation of neutral francium. J Phys B At Mol Opt Phys 1998;31:5301-14.

[18] Quinet P, Argante C, Fivet V, Terranova C, Yushchenko AV, Biémont E. Atomic data for radioactive elements Ra I, Ra II, Ac I and Ac II and application to their detection in HD 101065 and HR 465. Astron Astrophys 2007;474:307-14.

[19] Fivet V, Quinet P, Biémont E. Transition probabilities in complex ions: the case of americium. J Electron Spectrosc Relat Phenom 2007;156-158:255-8.

[20] Kramida A, Ralchenko Yu, Reader J, NIST ASD Team. NIST atomic spectra database (ver.5.1). Gaithersburg, MD: National Institute of Standards and Technology; 2013. 〈http://physics.nist.gov/asd〉.

[21] Charles GW. Spectra of ${ }^{208} \mathrm{Po}$ and the hyperfine structure of ${ }^{209} \mathrm{Po}$. J Opt Soc Am 1966;56:1292-7.

[22] Charles GW, Hunt DJ, Pish G, Timma DL. Preliminary description and analysis of the spectrum of polonium. J Opt Soc Am 1955;45:869-72.

[23] Mrozowski S. Multipole lines in the first spectrum of polonium I. J Opt Soc Am 1956;46:663.
[24] Cowan RD. The theory of atomic structure and spectra. Berkeley: University of California Press; 1981.

[25] Quinet P, Palmeri P, Biémont E, McCurdy MM, Rieger G, Pinnington $\mathrm{EH}$, et al. Experimental and theoretical lifetimes, branching fractions and oscillator strengths in Lu II. Mon Not R Astron Soc 1999;307:934-40.

[26] Quinet P, Palmeri P, Biémont E, Li ZS, Zhang ZG, Svanberg S. Radiative lifetime measurements and transition probability calculations in lanthanide ions. J Alloys Compd 2002;344:255-9.

[27] Biémont E, Quinet P. Recent advances in study of lanthanide atoms and ions. Phys Scr T 2003;105:38-54.

[28] Fraga S, Karwowski J, Saxena KMS. Handbook of atomic data. Amsterdam: Elsevier; 1976.

[29] Morillon C, Vergès J. Observation et classification du spectre d'arc du tellure (Te I) entre 3678 et $11761 \mathrm{~cm}^{-1}$. Phys Scr 1975;12:129-44. 